Proc. Indian Acad. Sei., Vol. 87 A (Mathematical Sciences-3), No. 9, September 1978, pp. 161-168, (C) printed in India

\title{
An integer arithmetic method to compute generalized matrix inverse and solve linear equations exactly
}

\author{
S K SEN and A A SHAMIM \\ Computer Centre, Indian Institute of Science, Bangalore 560012 \\ MS received 27 October 1977; revised 27 June 1978
}

\begin{abstract}
An algorithm that uses integer arithmetic is suggested. It transforms an $m \times n$ matrix to a diagonal form (of the structure of Smith Normal Form). Then it computes a reflexive generalized inverse of the matrix exactly and hence solves a system of linear equations error-free.
\end{abstract}

Keywords. Exact computation; integer arthematic; generalized matrix inverse; linear equations; Smith diagonai form.

\section{Introduction}

Whether a matrix $A$ over a complex field is singular square, or rectangular, it has always a generalized inverse ( $g$-inverse) over the (complex) field. The true inverse exists only when $A$ is nonsingular (i.e., a square matrix whose determinant is not zero). However, a $g$-inverse of an $m \times n$ matrix of rank $r$ involves considerable errors if the $r$ th order submatrices are near-singular. Further, the rank shown by the $g$-inverse may be less than the actual rank. In fact, identical are the pitfalls when a (square) near-singular matrix is inverted.

We present here a method that uses integer arithmetic to

(i) transform an $m \times n$ integral matrix to a Smith Diagonal Form (defined later) without requiring to compute the greatest common divisors (GCDs) of the matrix elements as required in computing certain $g$-inverses (Hurt and Waid [4], Ben-Israel and Greville [2]),

(ii) compute a reflexive $g$-inverse (Bowman and Burdet [3], Ben-Israel and Greville [2], Krishnamurthy and Sen [5]),

(iii) obtain a solution vector $x$ of $A x=b, b$ being 0 (null column vector) or not. Since any computing system can represent only the rational numbers, we can, without any loss of generality, assume the inputs (here the matrix $A$ and the right-hand-side column vector $b$ ) integral.

\section{Definitions}

\subsection{Integral vector and integral matrix}

Let (a) $K=$ the ring of integers $0, \pm 1, \pm 2, \ldots$,

(b) $K^{m}=$ the $m$ dimensional vector space over $K$, 
(c) $K^{m \times n}=$ the $m \times n$ matrices over $K$, and

(d) $K_{r}{ }^{m \times n}=$ the $m \times n$ matrices with rank $r$ over $K$.

Any element of $K^{m}$ is an integral vector. Any element of $K^{m \times n}$ is an integral matrix, and any element of $K_{r}^{m \times n}$ is an integral matrix of rank $r$.

\subsection{Elementary row and column operations}

A sequence of elementary row and column operations used here consists of

(a) Type 1. Interchanging two rows (columns),

(b) Type 2. Subtracting an integral multiple of one row (column) from another row (column), and

(c) Type 3. Replacing a row (column) by an integral multiple of the row (column).

\subsection{Elementary integral matrix}

Any nonsingular matrix $P \in K^{m \times m}$ (any nonsingular matrix $Q \in K^{n \times n}$ ) which when pre- (post-) multiply a given matrix $A \in K_{r}^{m \times n}$, produces a combination of types 1,2 , and 3 operations.

\subsection{Equivalent matrices}

Two matrices $A, S \in K^{m \times n}$ are equivalent over $K$ if there exist two elementary integral matrices $P \in K^{m \times m}$ and $Q \in K^{n \times n}$ such that $P A Q=S$.

\subsection{Smith diagonal form}

A matrix $S=\left(s_{i j}\right) \in K_{r}^{m \times n}$ is the Smith Diagonal Form (SDF) of $A \in K_{r}^{m \times n}$ if

(a) $s_{i l} \neq 0, i=1(1) r ;$ (b) $s_{i j}=0$ otherwise, and (c) $s_{t i}$ divides $s_{i+1}, i+1, i=1(1) r-1$.

\subsection{Generalized inverse of Smith diagonal form}

The $g$-inverse of SDF $S$ is the matrix $S^{+}=\left(s_{t_{J}}{ }^{+}\right) \in K_{r}{ }^{n \times m}$ if

(a) $s_{i i}{ }^{+}=s_{i i}{ }^{-1}, i=1(1) r$, and (b) $s_{i j}{ }^{+}=0$ otherwise.

\section{The method}

Let $A \in K_{r}^{m \times n}$ do not have any zero row or zero column.

Step 1. Computing SDF $S=\left(s_{i j}\right) \in K_{r}^{m \times n}$

(i) Make the elements at positions $(1,1),(2,2), \ldots$, nonzero using type 1 operations if any element at any of the positions $(1,1),(2,2), \ldots$, is zero. Otherwise go to step 1(ii). (ii) Multiply (type 3 operations) second, third,..., $m$ th rows by $p_{1}, p_{1} p_{2}, \ldots, p_{1} p_{2} \ldots p_{m-1}$, respectively, where $p_{i}$ is the element at position $(i, i)$ or $p_{i}$ is a number so that the 
elementary matrices $P_{1}, P_{2}, \ldots$, where $P=\ldots P_{2} P_{1}$, are integral (see step 2, and examples in section 6) or $p_{i}$ is a number so that $P$ is integral and the remainder in any division is zero.

(iii) Make zeros of all the elements below position $(1,1)$ in the first column using type 2 operations.

(iv) If the element at position $(2,2)$ is not zero then make zeros of all the elements below position $(2,2)$ in the second column using type 2 operations. Otherwise interchange $p_{2} p_{3} \ldots p_{s-1}$ times the second row and $1 /\left(p_{2} p_{3} \ldots p_{s-1}\right)$ times the $s$ th row whose second element is nonzero (types 1 and 3 operations).

Make zeros of all the elements below position $(2,2)$ in the second column (if these are not all zero). Continue the process for the elements at positions $(3,3),(4,4)$, etc.

Note. Steps 1(i) -1(iv) make the first $r$ rows non-null with all the elements below positions $(1,1),(2,2), \ldots$, etc. zero and other rows $((r+1)$ st to $m$ th rows $)$ null.

(v) Multiply second, third, ., rth columns by $q_{1}, q_{1} q_{2}, \ldots q_{1} q_{2} \ldots q_{r-1}$, respectively, where $q_{i}$ is the element at position $(i, i)$ or $q_{i}$ is a number so that the elementary matrices $Q_{1}, Q_{2}, \ldots$, where $Q=Q_{1} Q_{2}, \ldots$, are integral (see step 2 and examples in section 6) or $q_{i}$ is a number so that $Q$ is integral and the remainder in any division is zero. Multiply each of $(r+1)$ st, $(r+2)$ nd, $\ldots, n$th columns by $q_{1} q_{2} \ldots q_{r-1}$.

(vi) Make zeros of all the elements after position $(1,1)$ in the first row using type 2 operations.

(vii) If the element at position $(2,2)$ is not zero then make zeros of all the elements after position $(2,2)$ in the second row using type 2 operations. Otherwise interchange $q_{2} q_{3} \ldots q_{s-1}$ times the second column and $1 /\left(q_{2} q_{3} \ldots q_{s-1}\right)$ times the sth column whose second element is nonzero (types 1 and 3 operations) and then make zeros of all the elements after position $(2,2)$ in second row (if these are not all zero). Continue the process for the elements at positions $(3,3),(4,4)$, etc.

Note. Steps 1(i)-1(vii) give the SDF S. Also, in any divide operation denominator divides the numerator.

\section{Step 2. Computing $A^{-}$.}

(i) Compute the elementary matrix $P(Q)$ defined in sec. 2(iv), which is the product of all the elementary row (column) matrices, in the right order. Thus $P A Q=S$.

(ii) To obtain $A^{-}=Q S^{+} P$, compute

$$
\begin{aligned}
& (i, j) \text { th element of } a A^{-}=\sum_{k=1}^{r} q_{i k} p_{k j}\left(\alpha / s_{k k}\right), \\
& i=1(1) n, j=1(1) m \text { where } a=s_{r r} .
\end{aligned}
$$

Step 3. Computing solution vectors. To solve $A x=b, x$ being rational,

(i) compute $A \alpha A^{-} b$. If it is equal to $a b$, then solution exists. Otherwise, the system has no solution.

(ii) If $A a A^{-} b=a b$, then compute $a A^{-} b$.

$$
\text { Obtain } a x=a A^{-} b+a y-a A^{-} A y \text { for any } y \in K^{n} \text {. }
$$




\section{Remarks}

(i) Computing g-inverse of a rational matrix. Let $B$ be an $m \times n$ nonintegral rational matrix. Then $A=\beta B$ is integral. Hence $B^{-}=\beta A^{-}$.

(ii) Computing solution vector of a rational system. Let $C x=d$ be the nonintegral rational system. Then $E x=f$, where $E=\gamma C$ and $f=\gamma d$, is an integral system.

\section{Results}

The method follows from the theorem and corollary below.

Theorem. Let $A \in K_{r}^{m \times n}$. Then $A$ is equivalent over $K$ to an SDF $S \in K_{r}^{m \times n}$. The proof follows from the construction of $S$ described in step 1 of the method (Sec. 3).

Corollary. Let $P$ and $Q$ be elementary integral matrices and $P A Q=S$ be an SDF of $A \in K^{m \times n}$. Also, let $A^{-}=Q S^{+} P$. Then

$$
A A^{-} A=A, A^{-} A A^{-}=A^{-}
$$

Proof. $P A Q=S=S S^{+} S=P A Q S^{+} P A Q=P A A^{-} A Q$. Hence $A=A A^{-} A . \quad A^{-} A A^{-}$is proved similarly.

Note.

(i) Integrality condition. The $A^{-}$does not satisfy

$$
A^{-} A \in K^{n \times n} \text { and } A A^{-} \in K^{m \times m}
$$

if (nontrivial) type 3 operations are used.

(ii) Triangular matrices. If type 1 operations are not needed then $P$ and $Q$ will be lower and upper triangular matrices, respectively. In such a case step 2(ii) can be written as

To obtain $A^{-}=Q S^{+} P$, compute

$$
\begin{aligned}
& (i, j) \text { th element of } a A^{-}=\sum_{k=i}^{r} q_{i k} p_{k j}\left(\alpha / s_{k k}\right), i \geqslant j \\
& =\sum_{k=j}^{r} q_{i k} p_{k j}\left(a / s_{k k}\right), i<j \\
& i=1(1) r, j=1(1) r \text { where } a=s_{r r} \text {. }
\end{aligned}
$$

\section{Use of modular arithmetic}

For exact computation the modular arithmetic (Adegbeyeni and Krishnamurthy [1], Rao et al [6]) can be used only when the integer arithmetic (Sen and Shamim [7]), 
in a general purpose computing system, demands too long precision operands. The modular arithmetic offers the parallelism in computation but the total computation is about $u$ times the computation needed by the integer arithmetic, $u$ being the number of prime bases used.

\section{Examples}

$$
A=\left[\begin{array}{rrr}
2 & 3 & 5 \\
4 & 6 & 1 \\
3 & 5 & 10
\end{array}\right]
$$

Here $p_{1}=2, p_{2}=6, p_{3}=10$.

Step 1(ii). Multiply second and third rows by 2 and $2 \times 6$, respectively (type 3 ):

$$
P_{1} A=\left[\begin{array}{rrr}
1 & 0 & 0 \\
0 & 2 & 0 \\
0 & 0 & 12
\end{array}\right] \quad A=\left[\begin{array}{rrr}
2 & 3 & 5 \\
8 & 12 & 2 \\
36 & 60 & 120
\end{array}\right]
$$

Step 1(iii). To reduce the first column elements below position $(1,1)$ zero premultiply $P_{1} A$ by $P_{2}$ (type 2):

$$
P_{2} P_{1} A=\left[\begin{array}{rrr}
1 & 0 & 0 \\
-4 & 1 & 0 \\
-18 & 0 & 1
\end{array}\right] \quad P_{1} A=\left[\begin{array}{rrr}
2 & 3 & 5 \\
0 & 0 & -18 \\
0 & 6 & 30
\end{array}\right]
$$

Step 1(iv). The element at position $(2,2)$ is zero. So interchange $p_{2}$ times the second row and $\left(1 / p_{2}\right)$ times the third row (here $s=3$ ) whose second element is nonzero (types 1 and 3 ):

$$
P_{3} P_{2} P_{1} A=\left[\begin{array}{rrr}
1 & 0 & 0 \\
0 & 0 & 1 / 6 \\
0 & 6 & 0
\end{array}\right] \quad P_{2} P_{1} A=\left[\begin{array}{rrr}
2 & 3 & 5 \\
0 & 1 & 5 \\
0 & 0 & -108
\end{array}\right]
$$

Since the element below position $(2,2)$ in the second column is zero and $m=3$, we go to step $1(\mathrm{v})$.

Step 1(v). $q_{1}=2, q_{2}=1, q_{3}=-108$. Multiply second and third columns by $2,2 \times 1$, respectively (type 3 ):

$$
P_{3} P_{2} P_{1} A Q_{1}=P_{3} P_{2} P_{1} A\left[\begin{array}{lll}
1 & 0 & 0 \\
0 & 2 & 0 \\
0 & 0 & 2
\end{array}\right]=\left[\begin{array}{rrr}
2 & 6 & 10 \\
0 & 2 & 10 \\
0 & 0 & -216
\end{array}\right]
$$

Step 1(vi). Make zeros of all the elements after position $(1,1)$ in the first row (type 2):

$$
P_{3} P_{2} P_{1} A Q_{1} Q_{2}=P_{3} P_{2} P_{1} A Q_{1}=\left[\begin{array}{rrr}
1 & -3 & -5 \\
0 & 1 & 0 \\
0 & 0 & 1
\end{array}\right]=\left[\begin{array}{rrr}
2 & 0 & 0 \\
0 & 2 & 10 \\
0 & 0 & -216
\end{array}\right]
$$


Step 1(vii). Since the element at position $(2,2)$ is not zero, make zeros of all the elements (here one) after position $(2,2)$ in the second row (type 2 ):

$$
\begin{aligned}
& P_{3} P_{2} P_{1} A Q_{1} Q_{2} Q_{3}=P_{3} P_{2} P_{1} A Q_{1} Q_{2}\left[\begin{array}{rrr}
1 & 0 & 0 \\
0 & 1 & -5 \\
0 & 0 & 1
\end{array}\right]=\left[\begin{array}{rrr}
2 & 0 & 0 \\
0 & 2 & 0 \\
0 & 0 & -216
\end{array}\right]=S(\mathrm{SDF}) \\
& P=P_{3} P_{2} P_{1}=\left[\begin{array}{rrr}
1 & 0 & 0 \\
-3 & 0 & 2 \\
-24 & 12 & 0
\end{array}\right] \\
& Q=Q_{1} Q_{2} Q_{3}=\left[\begin{array}{rrr}
1 & -3 & 10 \\
0 & 2 & -10 \\
0 & 0 & 2
\end{array}\right], P A Q=S \\
& \alpha=s_{33}=-216 \\
& \alpha S^{+}=\left[\begin{array}{rrr}
-108 & 0 & 0 \\
0 & -108 & 0 \\
0 & 0 & 1
\end{array}\right]
\end{aligned}
$$

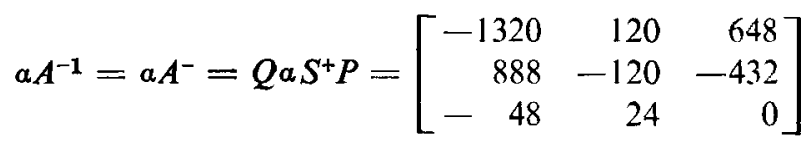

$$
\begin{aligned}
& A=\left[\begin{array}{rrrr}
-1 & 2 & 3 & 3 \\
2 & 5 & 6 & 3 \\
-5 & -8 & -9 & -3
\end{array}\right]
\end{aligned}
$$

Multiply second and third rows by 1 and $1 \times 5$, respectively (type 3 ):

$$
P_{1} A=\left[\begin{array}{lll}
1 & 0 & 0 \\
0 & 1 & 0 \\
0 & 0 & 5
\end{array}\right] \quad A \quad\left[\begin{array}{rrrr}
1 & 2 & 3 & 3 \\
2 & 5 & 6 & 3 \\
-25 & -40 & -45 & -15
\end{array}\right]
$$

To reduce the first column elements below position $(1,1)$ zero pre-multiply $P_{1} A$ by $P_{2}$ (type 2):

$$
P_{2} P_{1} A=\left[\begin{array}{lll}
1 & 0 & 0 \\
2 & 1 & 0 \\
0 & 0 & 5
\end{array}\right] \quad P_{1} A=\left[\begin{array}{rrrr}
-1 & 2 & 3 & 3 \\
0 & 9 & 12 & 9 \\
0 & -90 & -120 & -90
\end{array}\right]
$$

To reduce the second column element below position $(2,2)$ zero pre-multiply $P_{2} P_{1} A$ by $P_{3}$ (type 2 ):

$$
P_{3} P_{2} P_{1} A=\left[\begin{array}{rrr}
1 & 0 & -0 \\
0 & 1 & 0 \\
0 & 10 & 1
\end{array}\right] \quad P_{2} P_{1} A=\left[\begin{array}{rrrr}
-1 & 2 & 3 & 3 \\
0 & 9 & 12 & 9 \\
0 & 0 & 0 & 0
\end{array}\right]
$$


Multiply second, third, and fourth columns by $1,1 \times 9,1 \times 9$, respectively (type 3 ):

$$
P_{3} P_{2} P_{1} A Q_{1}=P_{3} P_{2} P_{1} A\left[\begin{array}{llll}
1 & 0 & 0 & 0 \\
0 & 1 & 0 & 0 \\
0 & 0 & 9 & 0 \\
0 & 0 & 0 & 9
\end{array}\right]=\left[\begin{array}{rrrr}
-1 & 2 & 27 & 27 \\
0 & 9 & 108 & 81 \\
0 & 0 & 0 & 0
\end{array}\right]
$$

To reduce the first row elements after position $(1,1)$ zero post-multiply $P_{3} P_{2} P_{1} A Q_{1}$ by $Q_{2}$ (type 2 ):

$$
P_{3} P_{2} P_{1} A Q_{1} Q_{2}=P_{3} P_{2} P_{1} A Q_{1}\left[\begin{array}{rrrr}
1 & 2 & 27 & 27 \\
0 & 1 & 0 & 0 \\
0 & 0 & 1 & 0 \\
0 & 0 & 0 & 1
\end{array}\right]=\left[\begin{array}{rrrr}
1 & 0 & 0 & 0 \\
0 & 9 & 108 & 81 \\
0 & 0 & 0 & 0
\end{array}\right]
$$

To reduce the second row elements after position $(2,2)$ zero post-multiply $P_{3} P_{2} P_{1} A Q_{1} Q_{2}$ by $Q_{3}$ (type 2 ):

$$
\begin{gathered}
P_{3} P_{2} P_{1} A Q_{1} Q_{2} Q_{3}=P_{3} P_{2} P_{1} A Q_{1} Q_{2}\left[\begin{array}{rrrr}
1 & 0 & 0 & 0 \\
0 & 1 & -12 & -9 \\
0 & 0 & 1 & 0 \\
0 & 0 & 0 & 1
\end{array}\right] \\
=\left[\begin{array}{rrrr}
-1 & 0 & 0 & 0 \\
0 & 9 & 0 & 0 \\
0 & 0 & 0 & 0
\end{array}\right]=S \\
P=P_{3} P_{2} P_{1}=\left[\begin{array}{rrr}
1 & \\
0 & 0 \\
1 & 0 \\
-5 & 10 & 5
\end{array}\right], Q \\
a=S_{22}=9, \alpha S^{+}=\left[\begin{array}{rrr}
-9 & 0 & 0 \\
0 & 1 & 0 \\
0 & 0 & 0 \\
0 & 0 & 0
\end{array}\right]=Q_{1} Q_{2} Q_{3}=\left[\begin{array}{rrrr}
1 & 2 & 3 & 9 \\
0 & 1 & -12 & -9 \\
0 & 0 & 9 & 0 \\
0 & 0 & 0 & 9
\end{array}\right] \\
a A^{-}=Q_{a} S^{+} P=\left[\begin{array}{rrr}
-5 & 2 & 0 \\
2 & 1 & 0 \\
0 & 0 & 0 \\
0 & 0 & 0
\end{array}\right]
\end{gathered}
$$

Note that $P A Q=S$, and $P$ and $Q$ are lower and upper triangular matrices, respectively since type 1 operations are not used.

$$
A a A^{-}=\left[\begin{array}{ccc}
9 & 0 & 0 \\
0 & 9 & 0 \\
9 & -18 & 0
\end{array}\right]
$$


If $b=\left(\begin{array}{lll}7 & 16 & -25\end{array}\right)^{t}$ then $A a A^{-} b=a b$ and hence solutions exist. If $b=\left(\begin{array}{lll}8 & 16 & -25\end{array}\right)^{t}$ then $A a A^{-} b \neq a b$ and hence solutions do not exist. For $b=(716-25)^{t}$ the solution vector $a x=\left(\begin{array}{llll}-3 & 30 & 0 & 0\end{array}\right)^{t}$.

\section{References}

[1] Adegbeyeni E O and Krishnamurthy E V 1977 Int. J. Systems Sci. 81181

[2] Ben-Israel A and Greville T N E 1974 Generalized Inverses: theory and applications (New York: Wiley-Interscience) p 94

[3] Bowman V J and Burdet C A 1974 SIAM J. Appl. Math. 26120

[4] Hurt M F and Waid C 1970 SIAM J. Appl. Math. 19547

[5] Krishnamurthy E V and Sen S K 1976 Computer-Based numerical Algorithms, (New Delhi: East-West)

[6] Rao T M,Subramanian K and Krishnamurthy E V 1976 SIAM J. Numer. Anal. 13155

[7] Sen S K and Shamim A A 1978 J. Indian Inst. Sci. 60111 